Dataset Paper

\title{
Investigation of the Working Parameters of a Single Magnetron of a Multiple Ion Cluster Source: Determination of the Relative Influence of the Parameters on the Size and Density of Nanoparticles
}

\author{
Manuel Ruano, Lidia Martínez, and Yves Huttel \\ Instituto de Ciencia de Materiales de Madrid, Consejo Superior de Investigaciones Científicas, C/ Sor Juana Inés de la Cruz 3, \\ 28049 Madrid, Spain
}

Correspondence should be addressed to Yves Huttel; huttel@icmm.csic.es

Received 4 March 2013; Accepted 2 April 2013

Academic Editors: B. Todorovic Markovic and T. Tsurui

Copyright (C) 2013 Manuel Ruano et al. This is an open access article distributed under the Creative Commons Attribution License, which permits unrestricted use, distribution, and reproduction in any medium, provided the original work is properly cited.

\begin{abstract}
Multiple Ion Cluster Source (MICS) is the new optimized route of a standard technique based on a sputtering gas aggregation source, the Ion Cluster Source. The single magnetron used in the standard Ion Cluster Source is replaced by three magnetrons inside the aggregation zone, and they are controlled individually in order to fabricate nanoparticles with the desired and tunable chemical composition. Apart from the working parameters of each magnetron, it is also reported that the relation between the working parameters of individual magnetrons is of prime importance for the control of both the size and density of the nanoparticles. The influences of fluxes of the sputtering gas applied to each magnetron, the total gas flux in the aggregation zone, the position in the aggregation zone of Ag magnetron, and the relative position of the magnetrons in the aggregation zone have been studied through the operation of one of the magnetrons loaded with a silver target.
\end{abstract}

\section{Introduction}

The technique of gas aggregation source is at the forefront of physical methods for the fabrication of nanoparticles (NPs) due to their industrial applications and importance in fundamental research. The fabrication of NPs using this method is based on the formation of a vapor made of elements that aggregate in order to synthesize the desired NPs. Among the different gas aggregation sources, we will focus on the one based on sputtering process due to its efficiency in the fabrication of NPs and to its ability to be used in combination with a quadrupole mass filter thanks to the fact that about $80 \%$ of the formed NPs are charged [1-4].

The standard ICS is composed by a single 2 -inch diameter magnetron that generates ions of a given material that aggregate in the so-called aggregation zone. The working parameters allow to control the size of the fabricated nanoparticles: (i) the power applied to the magnetron, (ii) the flux of sputtering gas (usually argon), (iii) the aggregation length (distance between the magnetron and the exit slit of the aggregation zone), (iv) the flux of an additional gas, $\Phi_{\text {Extra }}$ (usually helium), and (v) the aperture size between the aggregation zone and the deposition chamber where the NPs are collected over the desired surface. The flux of clusters or NPs that exit the ICS through the aperture is controlled by the deposition time. With this technique, the chemical composition of the NPs depends on the chemical composition of the target that is placed in the magnetron. It can be operated in ultrahigh vacuum (UHV) or vacuum conditions. Hence, the chemical purity of the nanoparticles can be guaranteed, which is mandatory for specific applications [5-7].

In order to overcome the limitation of a standard ICS that produces NPs with the same chemical composition as the one of the target, the design of the new Multiple Ion Cluster Source (MICS) has been recently patented [8]. The MICS that is now commercially available at Oxford Applied Research Ltd. [9] allows a fine-tuning of the chemical composition of the fabricated NPs [10]. The design is based on the 
TABLE 1: Working parameters in the Ion Cluster Source and Multiple Ion Cluster Sources equipments.

\begin{tabular}{lcc}
\hline Working parameters & $\begin{array}{c}\text { Ion Cluster } \\
\text { Source (ICS) }\end{array}$ & $\begin{array}{c}\text { Multiple Ion Cluster } \\
\text { Source (MICS) }\end{array}$ \\
\hline Flux of additional gas $\varphi_{\text {Extra }}$ & $\sqrt{ }$ & $\sqrt{ }$ \\
Aperture size & $\sqrt{ }$ & $\sqrt{ }$ \\
Power & $P$ & $P_{\mathrm{A}}, P_{\mathrm{B}}, P_{\mathrm{C}}$ \\
Flux of sputtering gas & $\varphi$ & $\varphi_{\mathrm{A}}, \varphi_{\mathrm{B}}, \varphi_{\mathrm{C}}$ \\
& & $\varphi_{T}$ \\
Aggregation length & $L$ & $L_{\mathrm{A}}, L_{\mathrm{B}}, L_{\mathrm{C}}$ \\
& & $L_{\mathrm{Rel}}$ \\
\hline
\end{tabular}

replacement of the single 2 -inch magnetron by 3 magnetrons of 1-inch diameter $(\mathrm{A}, \mathrm{B}, \mathrm{C})$ that are inserted in the aggregation zone. Each magnetron can be individually positioned inside the aggregation zone and is provided with its own sputtering gas feed (in our case Ar), cooling pipes, and electrical connections. The resulting MICS can be viewed as a triple standard ICS, as each magnetron can be operated like in a standard magnetron of an ICS (i.e., the power applied to each magnetron, each argon flux, and the individual position of the magnetron in the aggregation zone). Apart from this, MICS also presents common parameters with the standard ICS to control the NP size as a $\Phi_{\text {Extra }}$ entry and the aperture size. In addition to the mentioned working parameters, it has been observed that new parameters need to be taken into account in the fabrication of the NPs with the MICS: (a) the relative argon flux applied to each magnetron that defines the total argon flux $\Phi_{T}$, and (b) the relative position of each magnetron in the aggregation zone $\left(L_{\text {Rel }}\right)$. These parameters play an important role in the formation of the vapor generated in the aggregation zone and need to be understood for the fabrication of tunable nanostructures. The understanding of the influence of these new parameters is of great importance as they will be crucial when using combinations of two or three magnetrons for the fabrication of more complex systems. The mentioned working parameters are given in Table 1 in order to illustrate the differences between ICS and MICS options.

With the MICS, it is possible to make combinations of up to three materials in a controlled way to generate nanoparticles with the desired and controlled stoichiometry, without losing the size control. Recently, Martínez et al. reported the formation of bimetallic AuAg and trimetallic AuAgPd alloys [10]. Nevertheless, the use of more than one magnetron implies different gas pressures inside the aggregation zone and, therefore, changes in the final size of the NPs. In this dataset, we report on the influence of the two new parameters $\Phi_{T}$ and $L_{\text {Rel }}$ on the NP size when using one single magnetron.

\section{Methodology}

The MICS is schematically represented in Figure 1(a) in one of the configurations of the magnetrons employed in this work. The 3 magnetrons of 1-inch diameter are mounted on a 6-inch flange and inserted into the aggregation zone of an NC200U-B model nanocluster source from Oxford Applied Research Ltd. [9]. The fabrication of the magnetrons follows the original design of Professor Colino García from the Facultad de Ciencias del Medio Ambiente, Toledo, Spain [11]. The system was connected to a UHV chamber with a base pressure in the low $10^{-9}$ mbar. The clusters generated with the MICS were deposited on flat silicon wafers $\left(10 \times 10 \mathrm{~mm}^{2}\right)$ that were transferred into the chamber through a fast entry load lock. The working distance (distance between the exit slit of the MICS and the substrate placed into the manipulator of the chamber) was $\sim 200 \mathrm{~mm}$. Figure 1(b) presents a photograph of the MICS attached to the aggregation zone with their individual translators. In Figure 1(c), we present a picture of the three 1-inch diameter magnetrons before their introduction into the aggregation zone.

The magnetron used for this calibration was loaded with a silver (99.99\%) target. The other magnetrons, named B and C, were loaded with other targets but were not turned on for this study. The power applied to the $\operatorname{Ag}$ magnetron $\left(P_{\mathrm{Ag}}\right)$ was $8 \mathrm{~W}$ in all cases. The deposits have been performed with Ag, B, and $\mathrm{C}$ magnetrons positioned at different aggregation lengths ( $L_{\mathrm{Ag}}, L_{\mathrm{B}}$, and $L_{\mathrm{C}}$, resp.). B and $\mathrm{C}$ magnetrons were always placed behind Ag magnetron in order to avoid target contamination by Ag plasma (see Figure 1(a), where we illustrate the sputtered ions from the Ag target and their aggregation into NPs far from the other magnetrons). Apart from the aggregation length, the influences of Ar fluxes distribution in all of the magnetrons $\left(\Phi_{\mathrm{Ag}}, \Phi_{\mathrm{B}}\right.$, and $\left.\Phi_{\mathrm{C}}\right)$ and the total argon flux employed $\left(\Phi_{T}\right)$ were also studied. In the present work, the series of samples have been produced without a $\Phi_{\text {Extra }}$. At the indicated working distance $(\approx 200 \mathrm{~mm})$, the spot diameter of the area where the NPs landed was $\approx 40 \mathrm{~mm}$. The average size of the NPs of each deposit has been characterized by Atomic Force Microscopy (AFM) [10, 12, 13] using the Cervantes AFM System from Nanotec Electrónica S.L. [14]. The average size of the NPs is assumed to be identical to the average height of the NPs that is determined by AFM. This is reasonable taking into account that for similar deposition conditions, it has been found that the height and diameter are similar [12, 13]. The deposition rate in terms of number of $\mathrm{NP} \cdot \mu \mathrm{m}^{-2} \cdot \mathrm{s}^{-1}$ was also calculated from the AFM measurements.

Each mean value displayed in Figures 2, 3, 4, and 5 is the result of the size distribution study for a given nanoparticles deposit. Each deposit has been measured by AFM by recording images of scan areas of $1 \mu \mathrm{m} \times 1 \mu \mathrm{m}$. The number of AFM images required for the estimation of the size distribution is dependent on the coverage percentage and ranged from 3 to 7 images. The height of each nanoparticle in the AFM images is measured manually performing a profile, using ImageJ [1] and Gwyddion [2] softwares. In total, 67 AFM images were analyzed, and from these images, the height of 3604 nanoparticles was extracted. For each nanoparticles deposit, the fit of the nanoparticles size distribution was performed assuming a Galton or lognormal distribution [3], and the average (mean) size of each deposit was obtained. This protocol is detailed in [4].

In Figure 2, we have the average NP size and deposition rate as a function of $\Phi_{\mathrm{Ag}}$ (Table 2). The experimental $L_{\mathrm{Ag}}$ were $170 \mathrm{~mm}$ (red circles), $125 \mathrm{~mm}$ (blue squares), and $95 \mathrm{~mm}$ (green triangles). $L_{\mathrm{B}}=194 \mathrm{~mm}$ and $L_{\mathrm{C}}=203 \mathrm{~mm}$. For each 


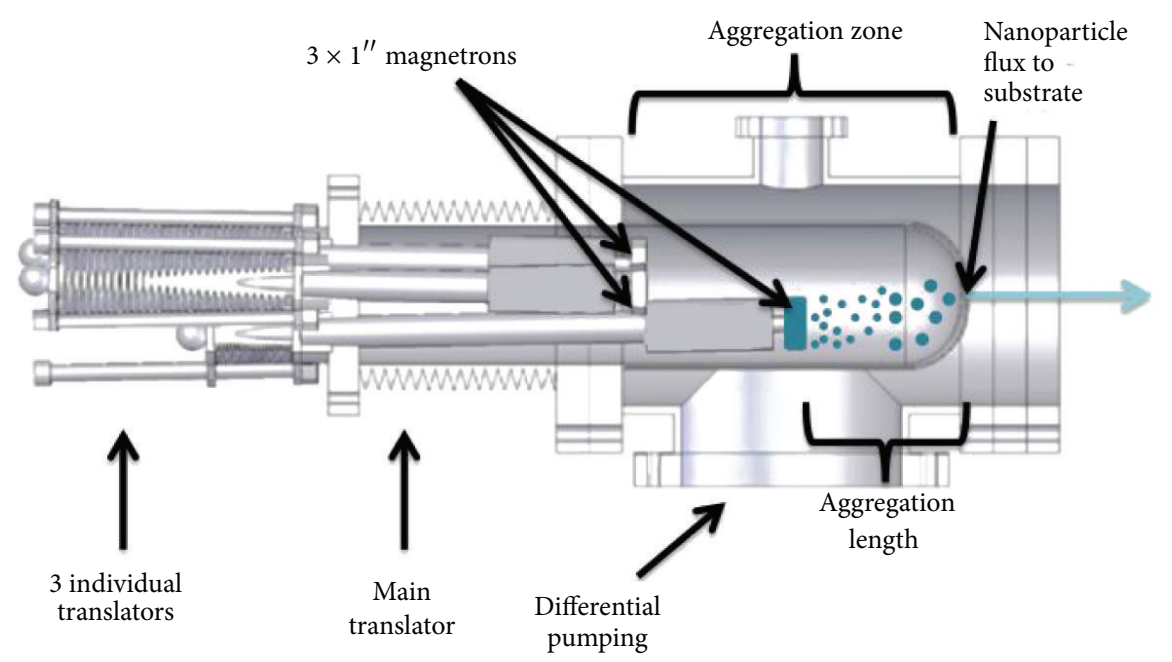

(a)

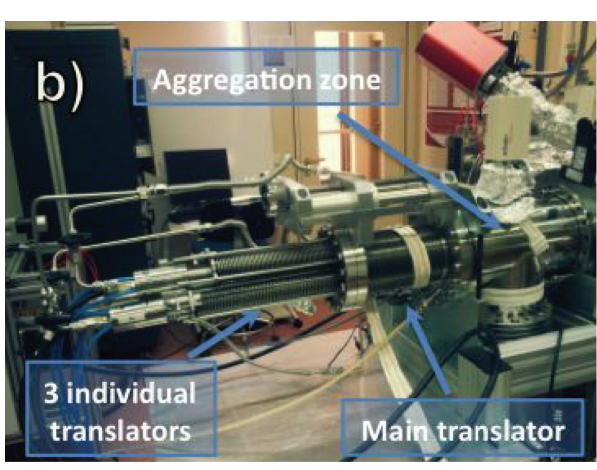

(b)

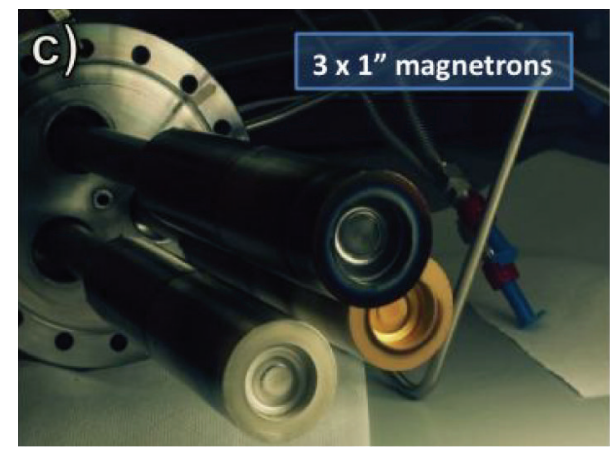

(c)

FIgure 1: Scheme of the Multiple Ion Cluster Source (MICS) in the configuration for using a single magnetron (a). Photograph of the MICS connected to the NC200U-B model ion cluster source (b). Photograph of the three individual 1-inch diameter magnetrons (c).

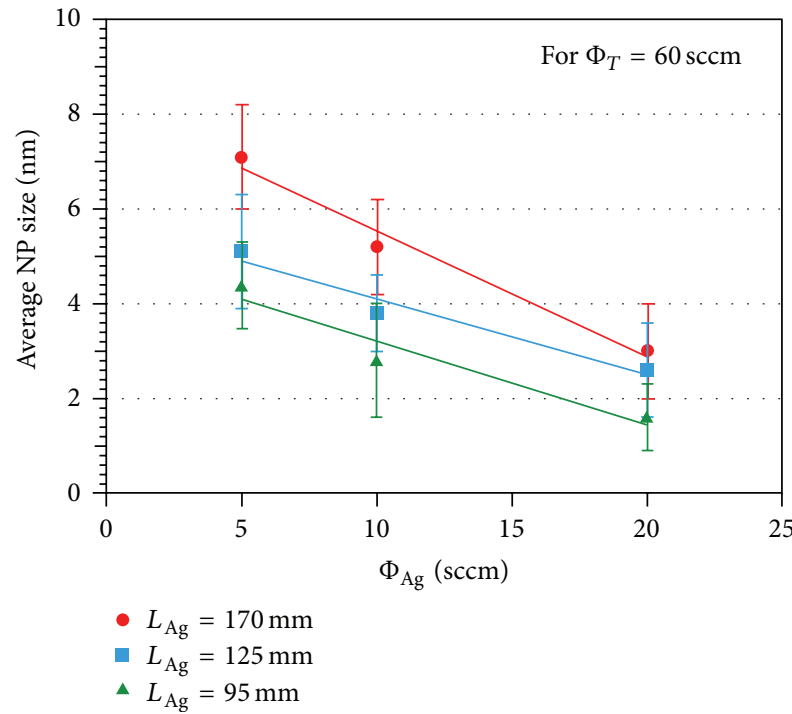

(a)

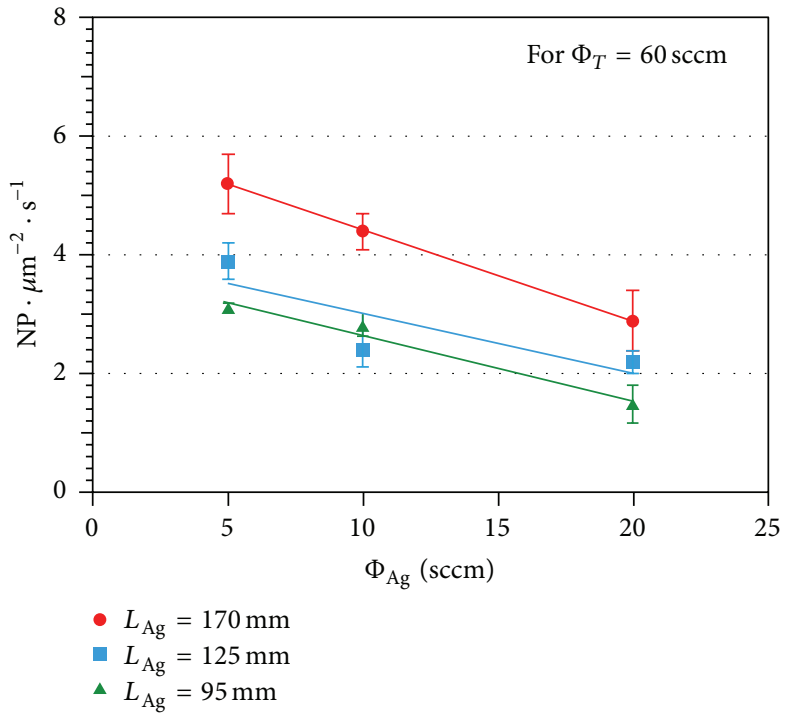

(b)

Figure 2: Average nanoparticle size (a) and deposition rate (b) as a function of the Argon flux applied to Ag magnetron, for different aggregation lengths of the Ag magnetron. The aggregation length is the distance between the magnetron and the exit slit of the aggregation zone. 


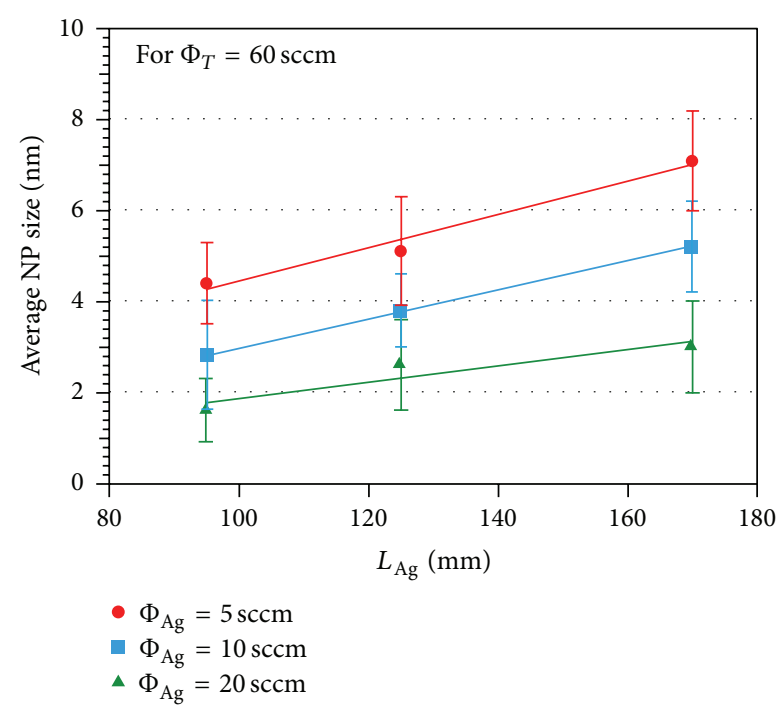

(a)

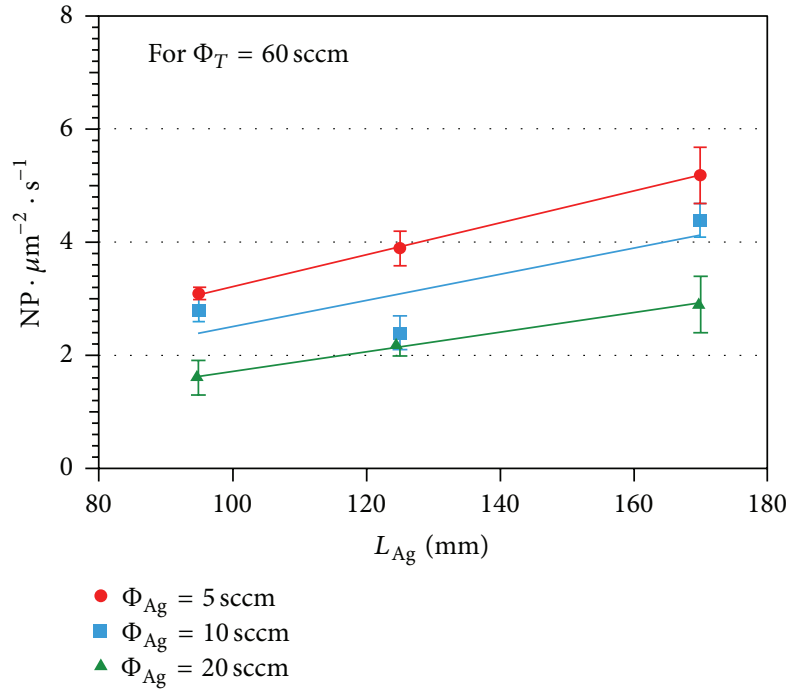

(b)

FIGURE 3: Average nanoparticle size (a) and deposition rate (b) as a function of aggregation length of the Ag magnetron, for different Ar fluxes applied to Ag magnetron. The aggregation length is the distance between the magnetron and the exit slit of the aggregation zone.

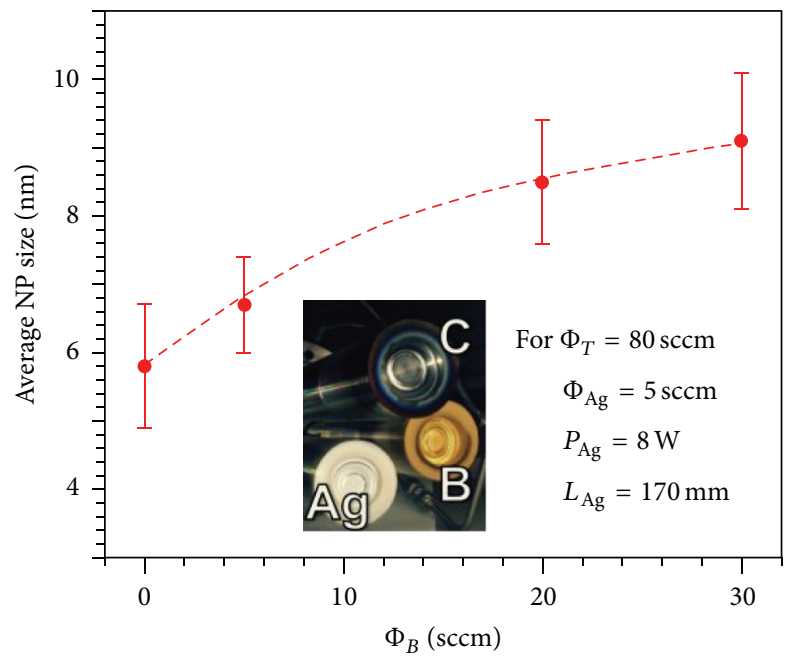

(a)

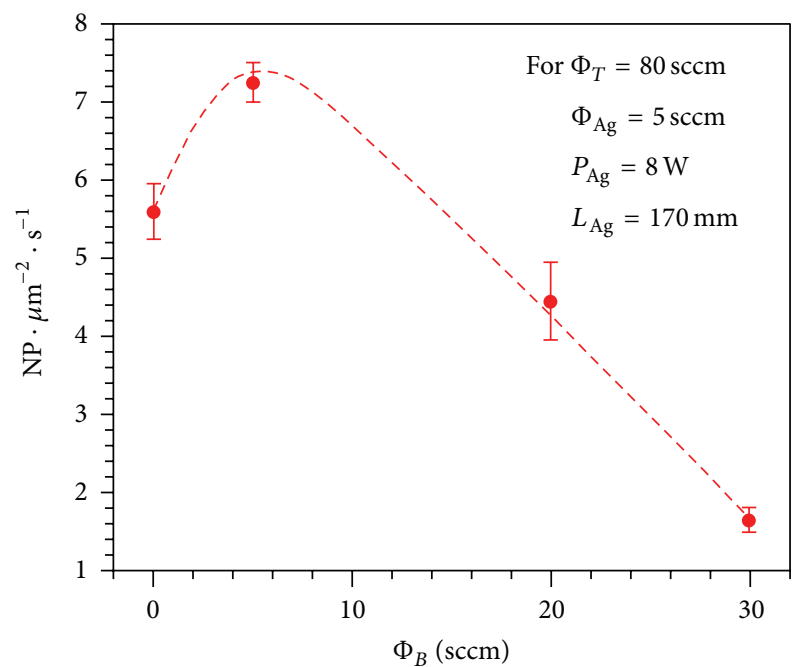

(b)

FIgURE 4: Average nanoparticle size (a) and deposition rate (b) as a function of the Argon flux applied to B magnetron, for a fixed total Ar flux and fixed Ar flux applied to Ag magnetron. The aggregation length is the distance between the magnetron and the exit slit of the aggregation zone.

$L_{\text {Ag }}$, three $\Phi_{\text {Ag }}$ were tested, $\Phi_{T}$ was kept constant at $60 \mathrm{sccm}$, and $\Phi_{\mathrm{B}}$ and $\Phi_{\mathrm{C}}$ always verified: $\Phi_{\mathrm{B}}=\Phi_{\mathrm{C}}=\left(\Phi_{T}-\Phi_{\mathrm{Ag}}\right) / 2$. The average NP size decreases linearly with $\Phi_{\mathrm{Ag}}$ for each $L_{\mathrm{Ag}}$ (Figure 2(a)). Moreover, this evolution is also observed in the case of the number of $\mathrm{NP} \cdot \mu \mathrm{m}^{-2} \cdot \mathrm{s}^{-1}$ as a function of $\Phi_{\text {Ag }}$ (Figure 2(b)). The studied AFM images and the size distribution data extracted from those AFM measurements, in order to obtain Figure 2 values, are detailed in Table 2.

In Figure 3, we have the average NP size and deposition rate as a function of $L_{\mathrm{Ag}}$ (Table 3 ). Three $\Phi_{\mathrm{Ag}}$ were applied: $5 \mathrm{sccm}$ (red circles), $10 \mathrm{sccm}$ (blue squares), and $20 \mathrm{sccm}$ (green triangles). $L_{\mathrm{B}}=194 \mathrm{~mm}$ and $L_{\mathrm{C}}=203 \mathrm{~mm} . \Phi_{T}$ was set at $60 \mathrm{sccm}$ and $\Phi_{\mathrm{B}}$ and $\Phi_{\mathrm{C}}$ verified: $\Phi_{\mathrm{B}}=\Phi_{\mathrm{C}}=$ $\left(\Phi_{T}-\Phi_{A g}\right) / 2$. Average NP size (Figure 3(a)) and deposition rate (Figure 3(b)) increase as a function of $L_{\mathrm{Ag}}$ for all $\Phi_{\mathrm{Ag}}$. At a given $L_{\mathrm{Ag}}$, the biggest NP size and highest deposition rate are obtained with the smallest $\Phi_{\mathrm{Ag}}$. The studied AFM images and the size distribution data extracted from those AFM measurements, in order to obtain Figure 3 values, are detailed in Table 3 .

In Figure 4, we have the average NP size and deposition rate as a function of $\Phi_{\mathrm{B}}$ at a fixed $\Phi_{T}$ (Table 4). $L_{\mathrm{Ag}}=170 \mathrm{~mm}$ and $L_{\mathrm{B}}=L_{\mathrm{C}}=195 \mathrm{~mm}$. $\Phi_{\mathrm{Ag}}$ was $5 \mathrm{sccm}$ and $\Phi_{T}$ was kept constant at $80 \mathrm{sccm}$. In that case, $\Phi_{\mathrm{B}}$ and $\Phi_{\mathrm{C}}$ were not equally 


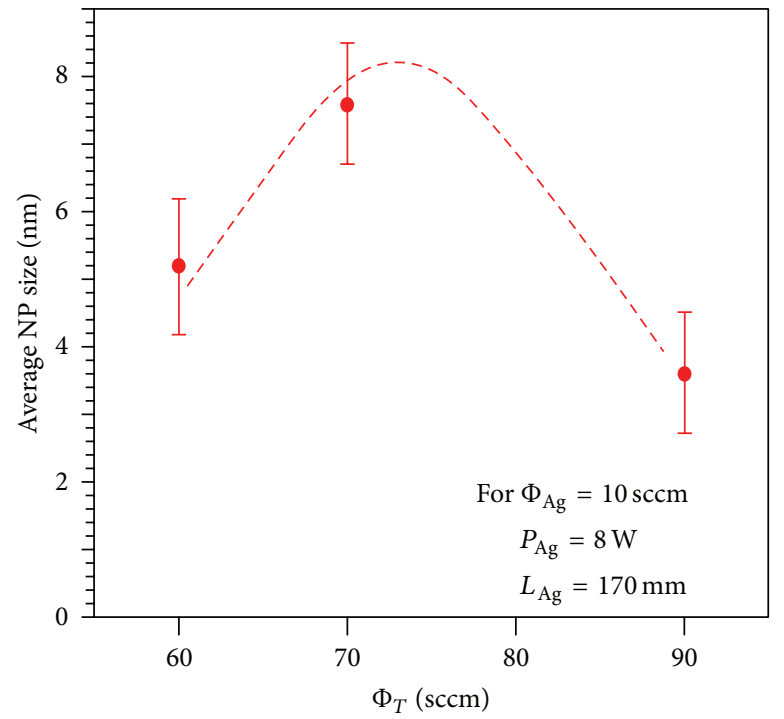

(a)

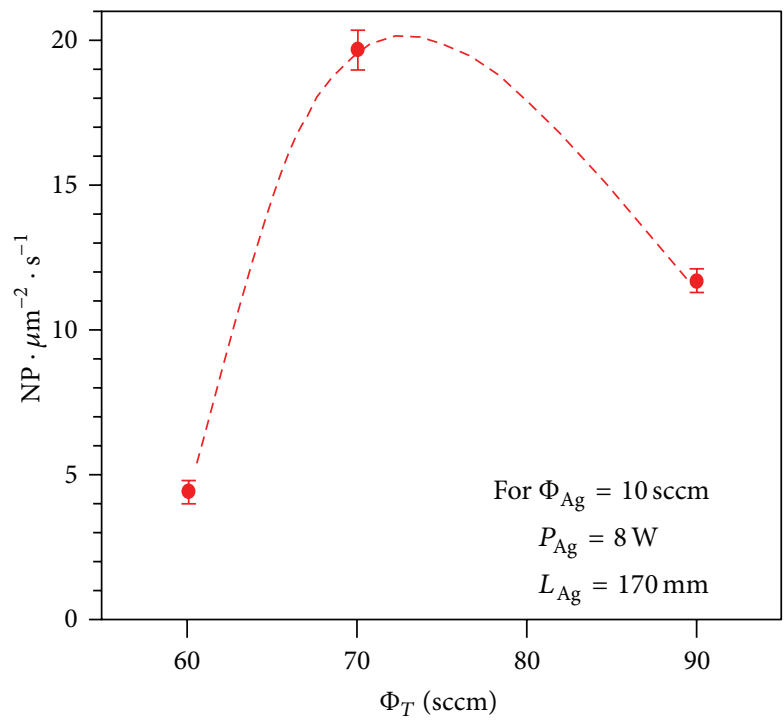

(b)

Figure 5: Average nanoparticle size (a) and deposition rate (b) as a function of the total Argon flux for a fixed Ar flux applied to Ag magnetron. The aggregation length is the distance between the magnetron and the exit slit of the aggregation zone.

Table 2: Row data from Figure 2 and details of the AFM images and the size distribution data associated to the Figure 2 values.

\begin{tabular}{|c|c|c|c|c|c|}
\hline \multicolumn{6}{|c|}{$\phi_{T}=60 \mathrm{sccm}$} \\
\hline$L_{\mathrm{Ag}}(\mathrm{mm})$ & $\varphi_{\mathrm{Ag}}(\mathrm{sccm})$ & Average NP size $(\mathrm{nm})$ & $\mathrm{NP} \cdot \mu \mathrm{m}^{-2} \cdot \mathrm{s}^{-1}$ & AFM images & Size distribution file \\
\hline \multirow{3}{*}{170} & 5 & $7.1 \pm 1.1$ & $5.2 \pm 0.5$ & Dataset Items 1-4 & Dataset Item 3 \\
\hline & 10 & $5.2 \pm 1.0$ & $4.4 \pm 0.3$ & Dataset Items 5-9 & Dataset Item 4 \\
\hline & 20 & $3.0 \pm 1.0$ & $2.9 \pm 0.5$ & Dataset Items 10-13 & Dataset Item 5 \\
\hline \multirow[t]{4}{*}{125} & 5 & $5.1 \pm 1.2$ & $3.9 \pm 0.3$ & Dataset Items 14-19 & Dataset Item 6 \\
\hline & 10 & $3.8 \pm 0.8$ & $2.4 \pm 0.3$ & Dataset Items 20-22 & Dataset Item 7 \\
\hline & 20 & $2.6 \pm 1.0$ & $2.2 \pm 0.2$ & Dataset Items 23-27 & Dataset Item 8 \\
\hline & 5 & $4.4 \pm 0.9$ & $3.1 \pm 0.1$ & Dataset Items 28-32 & Dataset Item 9 \\
\hline \multirow[t]{2}{*}{95} & 10 & $2.8 \pm 1.2$ & $2.8 \pm 0.2$ & Dataset Items 33-37 & Dataset Item 10 \\
\hline & 20 & $1.6 \pm 0.7$ & $1.5 \pm 0.3$ & Dataset Items 38-42 & Dataset Item 11 \\
\hline
\end{tabular}

balanced. $\Phi_{\mathrm{C}}$ verified: $\Phi_{\mathrm{c}}=\Phi_{T}-\Phi_{\mathrm{Ag}}-\Phi_{\mathrm{B}}$. The average NP size (Figure 4(a)) increases with $\Phi_{\mathrm{B}}$, whereas the deposition rate (Figure 4(b)) does not follow a similar evolution with $\Phi_{\mathrm{B}}$. Both dashed lines are guidelines for the eyes. The studied AFM images and the size distribution data extracted from those AFM measurements, in order to obtain Figure 4 values, are detailed in Table 4.

In Figure 5, we have the average NP size and deposition rate as a function of $\Phi_{\mathrm{T}}$ at a fixed $\Phi_{\mathrm{Ag}}$ (Table 5). $L_{\mathrm{Ag}}=$ $170 \mathrm{~mm}$ and $L_{\mathrm{B}}=L_{\mathrm{C}}=195 \mathrm{~mm}$. $\Phi_{\mathrm{Ag}}$ was $10 \mathrm{sccm}$. $\Phi_{\mathrm{B}}$ and $\Phi_{\mathrm{C}}$ verified: $\Phi_{\mathrm{B}}=\Phi_{\mathrm{C}}=\left(\Phi_{T}-\Phi_{\mathrm{Ag}}\right) / 2$. The evolution of the average NP size (Figure 5(a)) and the deposition rate (Figure 5(b)) as a function of $\Phi_{T}$ are similar. Both dashed lines are guidelines for the eyes. The studied AFM images and the size distribution data extracted from those AFM measurements, in order to obtain Figure 5 values, are detailed in Table 5.

\section{Dataset Description}

The dataset associated with this Dataset Paper consists of 17 items which are described as follows.

Dataset Item 1 (Images). Sixty-seven AFM images required for the estimation of the size distribution for nanoparticles deposits. Each deposit has been measured by AFM by recording images of scan areas of $1 \mu \mathrm{m} \times 1 \mu \mathrm{m}$. AFM images were analyzed, and from these images, the height of 3604 nanoparticles was extracted. For each nanoparticles deposit, the fit of the nanoparticles size distribution was performed.

Dataset Item 2 (3D Object Data). Sixty-seven STP files for the full content of Dataset Item 1 (Images) that allow the analysis of the images. 
TABLE 3: Row data from Figure 3 and details of the AFM images and the size distribution data associated to the Figure 3 values.

\begin{tabular}{|c|c|c|c|c|c|}
\hline & & & & & \\
\hline$L_{\mathrm{Ag}}(\mathrm{mm})$ & $\varphi_{\mathrm{Ag}}(\mathrm{sccm})$ & Average NP Size (nm) & $\mathrm{NP} \cdot \mu \mathrm{m}^{-2} \cdot \mathrm{s}^{-1}$ & AFM images & Size distribution file \\
\hline 170 & & $7.1 \pm 1.1$ & $5.2 \pm 0.5$ & Dataset Items 1-4 & Dataset Item 3 \\
\hline 125 & 5 & $5.1 \pm 1.2$ & $3.9 \pm 0.3$ & Dataset Items 14-19 & Dataset Item 6 \\
\hline 95 & & $4.4 \pm 0.9$ & $3.1 \pm 0.1$ & Dataset Items $28-32$ & Dataset Item 9 \\
\hline 170 & & $5.2 \pm 1.0$ & $4.4 \pm 0.3$ & Dataset Items 5-9 & Dataset Item 4 \\
\hline 125 & 10 & $3.8 \pm 0.8$ & $2.4 \pm 0.3$ & Dataset Items $20-22$ & Dataset Item 7 \\
\hline 95 & & $2.8 \pm 1.2$ & $2.8 \pm 0.2$ & Dataset Items 33-37 & Dataset Item 10 \\
\hline 170 & & $3.0 \pm 1.0$ & $2.9 \pm 0.5$ & Dataset Items 10-13 & Dataset Item 5 \\
\hline 125 & 20 & $2.6 \pm 1.0$ & $2.2 \pm 0.2$ & Dataset Items 23-27 & Dataset Item 8 \\
\hline 95 & & $1.6 \pm 0.7$ & $1.5 \pm 0.3$ & Dataset Items 38-42 & Dataset Item 11 \\
\hline
\end{tabular}

TABle 4: Row data from Figure 4 and details of the AFM images and the size distribution data associated to the Figure 4 values.

\begin{tabular}{|c|c|c|c|c|c|c|}
\hline \multicolumn{7}{|c|}{$\varphi_{T}=80 \mathrm{sccm}$} \\
\hline$L_{\mathrm{Ag}}(\mathrm{mm})$ & $\varphi_{\mathrm{Ag}}(\mathrm{sccm})$ & $\varphi_{\mathrm{B}}(\mathrm{sccm})$ & $\begin{array}{l}\text { Average NP } \\
\text { size (nm) }\end{array}$ & $\mathrm{NP} \cdot \mu \mathrm{m}^{-2} \cdot \mathrm{s}^{-1}$ & AFM images & Size distribution file \\
\hline \multirow{4}{*}{170} & \multirow{4}{*}{5} & 0 & $5.8 \pm 0.9$ & $5.6 \pm 0.4$ & Dataset Items 43-46 & Dataset Item 12 \\
\hline & & 5 & $6.7 \pm 0.7$ & $7.3 \pm 0.3$ & Dataset Items $47-50$ & Dataset Item 13 \\
\hline & & 20 & $8.5 \pm 0.9$ & $4.4 \pm 0.5$ & Dataset Items 51-54 & Dataset Item 14 \\
\hline & & 30 & $9.1 \pm 1.0$ & $1.6 \pm 0.2$ & Dataset Items 55-59 & Dataset Item 15 \\
\hline
\end{tabular}

Dataset Item 3 (Table). Size distribution data extracted from images 1-4 in Dataset Item 1.

Column 1: NP Size (nm)

Column 2: Number of Events

Dataset Item 4 (Table). Size distribution data extracted from images 5-9 in Dataset Item 1.

Column 1: NP Size (nm)

Column 2: Number of Events

Dataset Item 5 (Table). Size distribution data extracted from images $10-13$ in Dataset Item 1.

Column 1: NP Size (nm)

Column 2: Number of Events

Dataset Item 6 (Table). Size distribution data extracted from images 14-19 in Dataset Item 1.

Column 1: NP Size (nm)

Column 2: Number of Events

Dataset Item 7 (Table). Size distribution data extracted from images 20-22 in Dataset Item 1.

Column 1: NP Size (nm)

Column 2: Number of Events
Dataset Item 8 (Table). Size distribution data extracted from images 23-27 in Dataset Item 1.

Column 1: NP Size (nm)

Column 2: Number of Events

Dataset Item 9 (Table). Size distribution data extracted from images 28-32 in Dataset Item 1.

Column 1: NP Size (nm)

Column 2: Number of Events

Dataset Item 10 (Table). Size distribution data extracted from images 33-37 in Dataset Item 1.

Column 1: NP Size (nm)

Column 2: Number of Events

Dataset Item 11 (Table). Size distribution data extracted from images 38-42 in Dataset Item 1.

Column 1: NP Size (nm)

Column 2: Number of Events

Dataset Item 12 (Table). Size distribution data extracted from images 43-46 in Dataset Item 1.

Column 1: NP Size (nm)

Column 2: Number of Events 
Table 5: Row data from Figure 5 and details of the AFM images and the size distribution data associated to the Figure 5 values.

\begin{tabular}{|c|c|c|c|c|c|c|}
\hline$L_{\mathrm{Ag}}(\mathrm{mm})$ & $\varphi_{\mathrm{Ag}}(\mathrm{sccm})$ & $\varphi_{T}(\mathrm{sccm})$ & $\begin{array}{c}\text { Average NP } \\
\text { size }(\mathrm{nm})\end{array}$ & $\mathrm{NP} \cdot \mu \mathrm{m}^{-2} \cdot \mathrm{s}^{-1}$ & AFM images & Size distribution file \\
\hline \multirow{3}{*}{170} & \multirow{3}{*}{10} & 60 & $5.2 \pm 1.0$ & $4.4 \pm 0.4$ & Dataset Items 5-9 & Dataset Item 4 \\
\hline & & 70 & $7.6 \pm 0.9$ & $19.7 \pm 0.7$ & Dataset Items 60-63 & Dataset Item 16 \\
\hline & & 90 & $3.6 \pm 0.9$ & $11.7 \pm 0.4$ & Dataset Items 64-67 & Dataset Item 17 \\
\hline
\end{tabular}

Dataset Item 13 (Table). Size distribution data extracted from images 47-50 in Dataset Item 1.

Column 1: NP Size (nm)

Column 2: Number of Events

Dataset Item 14 (Table). Size distribution data extracted from images 51-54 in Dataset Item 1.

\section{Column 1: NP Size (nm) \\ Column 2: Number of Events}

Dataset Item 15 (Table). Size distribution data extracted from images 55-59 in Dataset Item 1.

\section{Column 1: NP Size (nm)}

Column 2: Number of Events

Dataset Item 16 (Table). Size distribution data extracted from images 60-63 in Dataset Item 1.

Column 1: NP Size (nm)

Column 2: Number of Events

Dataset Item 17 (Table). Size distribution data extracted from images 64-67 in Dataset Item 1.

\section{Column 1: NP Size (nm) \\ Column 2: Number of Events}

\section{Concluding Remarks}

MICS is characterized by the individual control of three magnetrons inserted in a gas aggregation zone. This dataset presents the calibration of one single magnetron of the MICS and highlights the singularities of this fabrication technique in comparison to standard ICS. We have focused on the influence of new working parameters on the average nanoparticle size and deposition rate. The possibility to insert argon through the other magnetrons modifies the total argon flux in the aggregation zone and, thus, the size of the fabricated NPs. In addition, the relative position of the magnetrons inside the aggregation zone also influences the final NP size. The control of these two new working parameters allows the fabrication of nanoparticles in a tunable way that in turns open the route for the generation of new nanoparticles.

\section{Dataset Availability}

The dataset associated with this Dataset Paper is dedicated to the public domain using the CC0 waiver and is available at http://dx.doi.org/10.7167/2013/597023/dataset.

\section{Acknowledgments}

This work was supported by the Spanish Ministerio de Ciencia e Innovación and Comisión Interministerial para la Ciencia y la Tecnología - (CICYT) under contracts MAT200806765-C02-02, MAT2011-29194-C02-02, and CSD2007-00041 (NANOSELECT). Manuel Ruano acknowledges the FPI program for financial support. The Consejo Superior de Investigaciones Científicas is also acknowledged under contract PIE $201160 \mathrm{E} 085$.

\section{References}

[1] H. Haberland, M. Karrais, M. Mall, and Y. Thurner, "Thin films from energetic cluster impact: a feasibility study," Journal of Vacuum Science \& Technology A, vol. 10, no. 5, pp. 3266-3271, 1992.

[2] H. Haberland, M. Mall, M. Moseler, Y. Qiang, T. Reiners, and Y. Thurner, "Filling of micron-sized contact holes with copper by energetic cluster impact," Journal of Vacuum Science \& Technology A, vol. 12, no. 5, p. 2925, 1994.

[3] S. H. Baker, S. C. Thornton, A. M. Keen et al., "The construction of a gas aggregation source for the preparation of mass-selected ultrasmall metal particles," Review of Scientific Instruments, vol. 68, no. 4, pp. 1853-1857, 1997.

[4] S. H. Baker, S. C. Thornton, K. W. Edmonds, M. J. Maher, C. Norris, and C. Binns, "The construction of a gas aggregation source for the preparation of size-selected nanoscale transition metal clusters," Review of Scientific Instruments, vol. 71, no. 8, pp. 3178-3183, 2000.

[5] J. Schmelzer Jr., S. A. Brown, A. Wurl, M. Hyslop, and R. J. Blaikie, "Finite-size effects in the conductivity of cluster assembled nanostructures," Physical Review Letters, vol. 88, Article ID 226802, 4 pages, 2002.

[6] R. Russo, A. Cianchi, Y. H. Akhmadeev et al., "UHV arc for high quality film deposition," Surface and Coatings Technology, vol. 201, no. 7, pp. 3987-3992, 2006.

[7] R. Russo, L. Catani, A. Cianchi, S. Tazzari, and J. Langner, "High quality superconducting niobium films produced by an ultrahigh vacuum cathodic arc," Superconductor Science and Technology, vol. 18, no. 7, pp. L41-L44, 2005.

[8] E. L. Román García, L. Martínez Orellana, M. Díaz Lagos, and Y. Huttel, Spanish Patent P201030059, PCT/ES2011/070032, 2010.

[9] Oxford Applied Research, http://www.oaresearch.co.uk/. 
[10] L. Martínez, M. Díaz, E. Román, M. Ruano, P. D. Llamosa, and Y. Huttel, "Generation of nanoparticles with adjustable size and controlled stoichiometry: recent advances," Langmuir, vol. 28, no. 30, pp. 11241-11249, 2012.

[11] J. M. Colino García, "Unidad de pulverización catódica de blancos circulares," Spanish Patent P200900929, 2009.

[12] M. Ruano, M. Díaz, L. Martínez et al., "Matrix and interaction effects on the magnetic properties of Co nanoparticles embedded in gold and vanadium," Physical Chemistry Chemical Physics, vol. 15, no. 1, pp. 316-329, 2013.

[13] M. Díaz, L. Martínez, M. Ruano et al., "Morphological, structural, and magnetic properties of Co nanoparticles in a silicon oxide matrix," Journal of Nanoparticle Research, vol. 13, no. 10, pp. 5321-5333, 2011.

[14] http://www.nanotec.es/. 

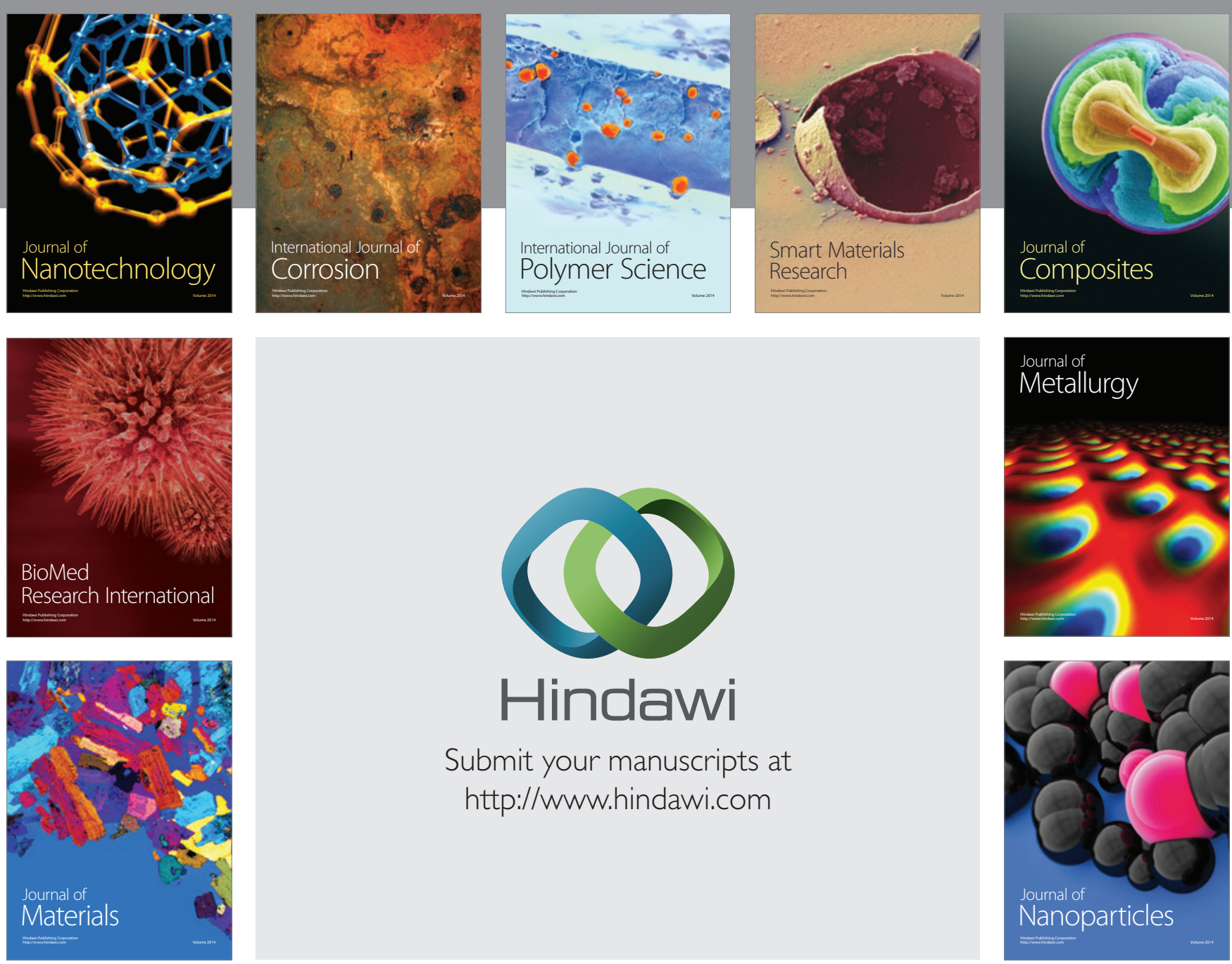

Submit your manuscripts at http://www.hindawi.com
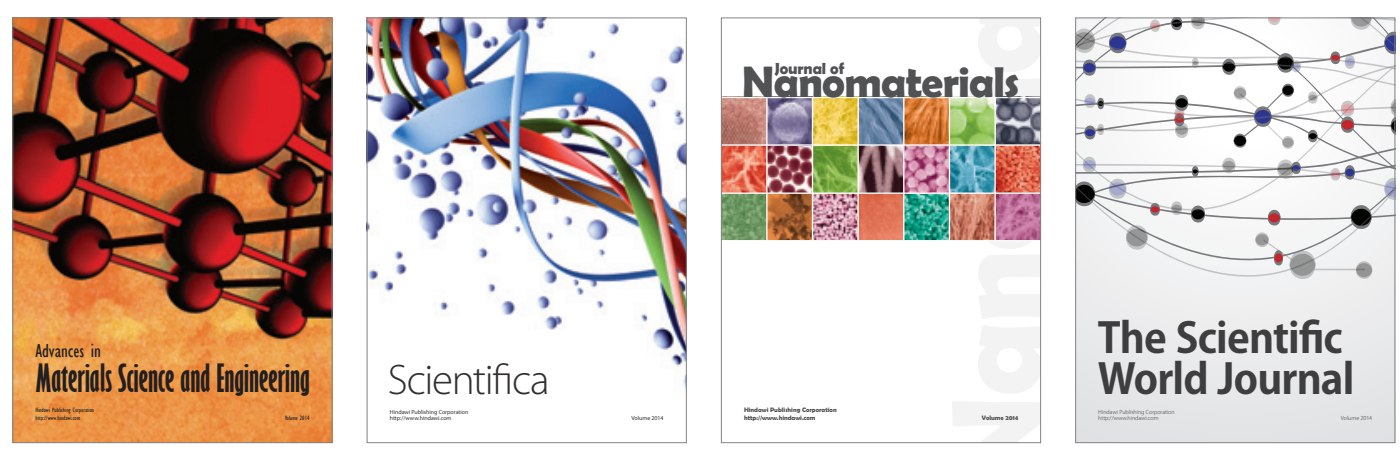

\section{The Scientific World Journal}
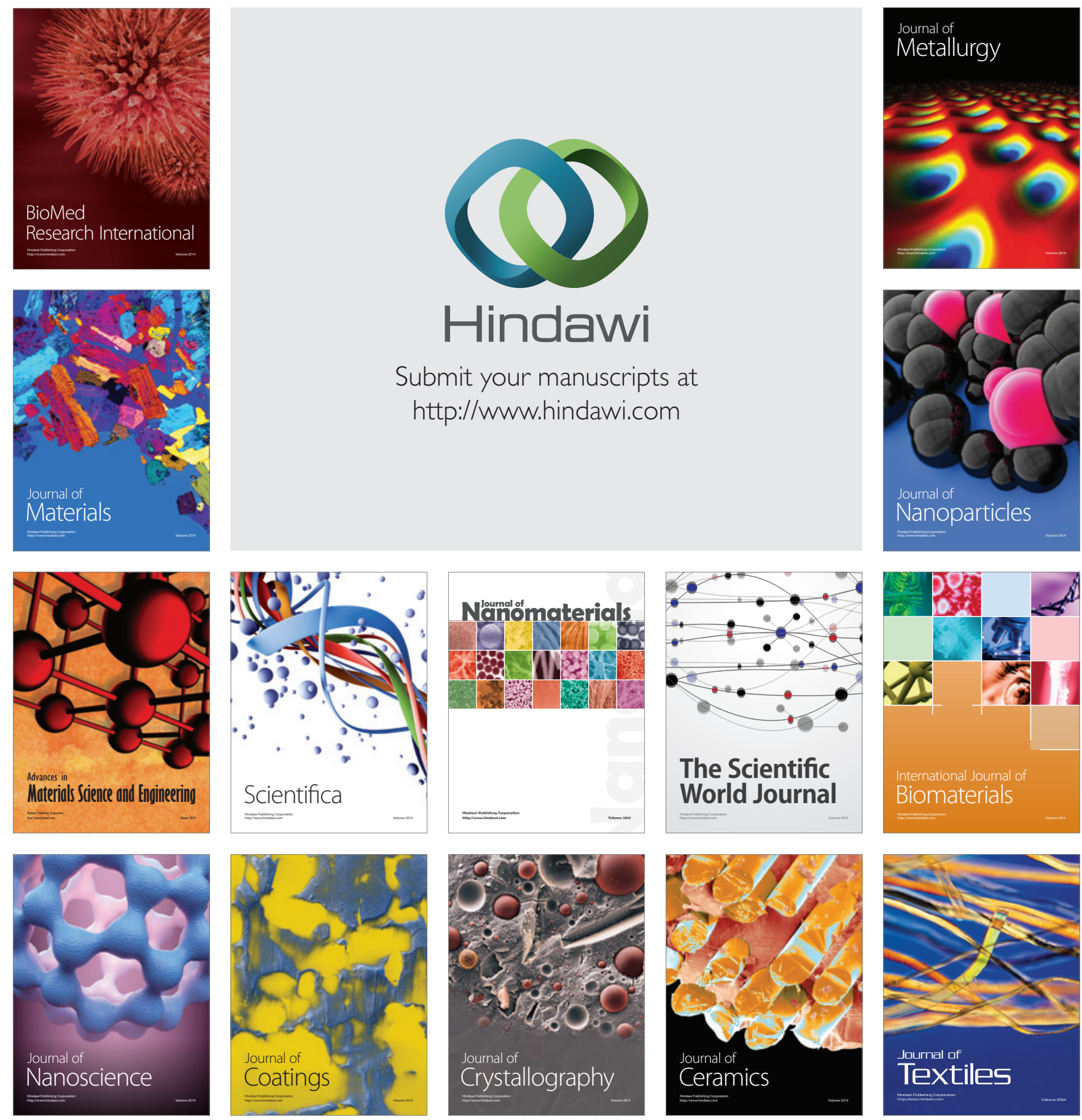